\title{
Oportunidades desiguales: empresas y Estado en conflictos sobre la minería en Chile
}

\section{Unequal opportunities: companies and the State in contention over mining in Chile}

\author{
Bettina Schorr ${ }^{1}$
}

\begin{abstract}
Resumen
Este artículo analiza variaciones significativas entre dos conflictos en torno a la minería en Chile con respecto al grado, la escala y los resultados de las contiendas: los conflictos en Chañaral, donde la empresa estatal Codelco opera, y en el valle del Huasco (Pascua Lama), donde una empresa privada transnacional está a cargo. En el primer caso la oposición contra la minería es baja y aislada, y el Estado no responde a las demandas locales. En contraste, el proyecto Pascua Lama motivó protestas a gran escala, obtuvo apoyo nacional e internacional y logró que el Estado actuara en beneficio de las demandas locales. Tres factores explican estas diferencias: primero, el contexto económico local, es decir, la dependencia que un determinado lugar tiene de la industria minera afecta el grado de movilización; segundo, la presencia de la empresa pública reduce tanto la disposición general de oponerse a la actividad minera como las oportunidades de multiplicar las escalas del conflicto. Además, el Estado tiende a proteger a las empresas públicas que proporcionan ingresos fiscales importantes. Por último, el origen de la empresa (nacional-transnacional) influye en la escala, así como en los resultados, de los conflictos mineros.
\end{abstract}

Palabras claves: conflictos mineros, compañías públicas, relaciones transnacionales, industria minera en Chile. movilización social.

\begin{abstract}
This article examines significant variations between two conflicts over mining in Chile regarding the degree, the scale and the outcomes of contention: the conflicts in Chañaral where the public company Codelco operates and in the Huasco Valley (Pascua Lama) where a transnational company is in charge. While the former suffers widespread contamination caused by mining activities, opposition against mining remains low and isolated and the state barely responds to local claims. In contrast, the mining project of Pascua Lama motivated large-scale protests, reached national and international support and managed that state agencies partly acted on behalf of local claims. Three factors explain these differences: First, the local economic context, i.e. the dependency of a given place on the mining industry affects the degree and shape of contention; second, the presence of the public company, which both reduces the overall disposition to contest its activities as well as the opportunities to 'scaleup' contention. Moreover, state authorities tend to protect public companies that provide significant fiscal incomes; Lastly, the origin of the company (national - transnational) shapes the scale as well as the outcomes of contention over mining.
\end{abstract}

Keywords: contention over mining, public companies, transnational relations, mining industry in Chile, social mobilization.

Recibido: 4 agosto 2016. Aceptado: 14 marzo 2017.

1 Freie Universität Berlin, Instituto de Estudios Latinoamericanos, ALEMANIA.

Email: bettina.schorr@fu-berlin.de 


\section{Introducción}

América Latina registró desde la década de los noventa un crecimiento importante en el sector minero (Bridge, 2004). Ello se debe principalmente a dos factores: la creciente demanda de las economías emergentes, sobre todo de China y de la India, y las reformas neoliberales introducidas en toda la región para combatir las hiperinflaciones y graves endeudamientos públicos generados en las décadas anteriores (Bridge, 2004; Bebbington y Bury, 2013). En el ámbito de la minería (como en otros sectores), la estrategia neoliberal impuso privatizaciones, nuevas regulaciones para atraer inversiones transnacionales y una serie de incentivos sobre todo tributarios para las empresas. Aunque económicamente importante para los Estados que reciben los ingresos en términos de impuestos y regalías (Burchardt y Dietz, 2014), la minería suele representar un costo muy alto para los lugares donde opera: degradación ambiental y dańos sobre la salud pública de poblados cercanos por el uso de agentes tóxicos, escasez de agua y tierra, reasentamientos humanos y migración forzosa, para nombrar los más importantes (Gudynas, 2009; Alimonda, 2011; Bebbington, 2012; Göbel y Ulloa, 2014; Dietz y Engels, 2016; para Chile ver Barton, Bustos y Prieto, 2015). En consecuencia, se multiplicaron en toda la región las movilizaciones sociales en contra de proyectos o instalaciones mineras, redundando en un panorama de conflictividad social importante.

Mientras que el número global de conflictos sociales en torno a la minería aumentó, se puede constatar una gran variación tanto entre países como dentro de Estados en cuanto a la intensidad de la movilización social, la escala de conflictos y las reacciones de las autoridades estatales. El valle del Huasco y la comunidad de Chañaral, ambas situadas en la región de Atacama en el norte de Chile, ejemplifican esta variación dentro del mismo contexto político institucional: son dos lugares en donde la minería y sus efectos son bastante controversiales. Sin embargo, aunque ambos experimentan conflictos duraderos, existen diferencias significativas.

Pascua Lama es el nombre de un gran proyecto binacional de minería enfocado en la extracción de oro en la frontera chileno-argentina al sur del de- sierto de Atacama. Por el lado chileno los minerales se extienden hasta la zona de Copiapó y la provincia de Huasco. En Argentina, el oro está localizado en la Provincia de San Juan. En Chile, Pascua Lama se ubica dentro de los conflictos mineros de más larga duración en los últimos tiempos. ${ }^{2}$ Comenzó en el año 2000 cuando la multinacional canadiense Barrick Gold Corp. anunció su plan de invertir en la que se convertiría en una de las minas de oro más grandes del planeta. Por los últimos 15 ańos, tanto el proyecto como la compañía han sido resistidos por diferentes grupos locales, basados en preocupaciones ecológicas y de posible escasez de agua debido al intenso consumo esperado por parte de la minera. Activistas locales lograron organizar protestas masivas, obtuvieron apoyo local, nacional e internacional para su causa y ganaron acceso a instituciones públicas nacionales. La constante resistencia local combinada con sanciones de parte de instituciones públicas y los bajos precios globales del oro dieron lugar a la más reciente suspensión del proyecto.

Por su parte la comunidad de Chañaral está ubicada en la costa del Pacífico, hacia el norte del valle del Huasco. Desde mediados del siglo XX, dos minas localizadas al este de Chañaral han depositado sus desechos mineros tóxicos en el río Salado, que se une al océano Pacífico en la bahía de Chañaral: Potrerillos, que fue cerrada en 1959, y luego la mina El Salvador (operativa en la actualidad). Inicialmente esta última fue propiedad de la compañía estadounidense Andes Copper Corporation. En 1972 fue nacionalizada y pasó luego a manos de la estatal Codelco (Corporación del Cobre), la cual continuó con los procedimientos nocivos hasta 1988 , cuando un fallo del Tribunal Constitucional de Chile obligó a construir un dique de contención (Vergara, 2011).

Hasta ahora, más de 350 millones de toneladas de desechos mineros tóxicos han alcanzado la bahía de Chańaral (Cortés, 2014). Como consecuencia, el mar se retiró dejando un paisaje lunar de arenas blancas lleno de agentes tóxicos. Y hasta el día de hoy ocurren esporádicamente escapes de materiales

2 Para una visión general cronológica del conflicto sobre Pascua Lama, ver Urquidi (2010), Karmy y Salinas (2009) y OLCA (2011). 
tóxicos. ${ }^{3}$ Además de esto, en años recientes, la polución ha afectado al parque nacional Pan de Azúcar, al norte de la ciudad. Numerosos estudios técnicos privados han confirmado el impacto negativo de esta contaminación sobre el ecosistema local y sobre la salud humana (Cortés, 2014).

Por ya más de tres décadas, grupos de activistas han intentado movilizar a sus conciudadanos para enfrentar esta situación. Envueltos en disputas con el Estado chileno y Codelco, apuntan principalmente a dos objetivos: en primer lugar, detener la mala práctica de la compañía de verter sus relaves en el mar (lo que fue alcanzado gracias al mandato del Tribunal Constitucional en 1988) y en segundo lugar, recibir una indemnización y forzar al Estado y a la compañía a reparar los daños ambientales derivados. Sin embargo, en comparación con sus vecinos en la región de Huasco, los activistas en Chañaral no lograron altos niveles de protesta en contra de la minera. Tampoco alcanzaron a generar apoyo nacional, ganar acceso institucional o conectar su causa a redes transnacionales. Por último, más allá del fallo del Tribunal Constitucional, faltan respuestas por parte de las autoridades a estas demandas.

Obviamente, estas dos localidades que en el mismo país y hasta en la misma región (Atacama) enfrentan una desigualdad de oportunidades para la expresión de su voz (en el sentido del grado y la escala de movilización) e impacto (en el sentido de lograr que las autoridades actúen en nombre de sus demandas): mientras los activistas en Pascua Lama fueron capaces de multiplicar las arenas y niveles donde se disputa su causa y, por último, manejarse para activar a los organismos estatales, los activistas en Chañaral aún no lo han logrado, aunque hay un grupo de ecologistas intentándolo sin descanso.

El objetivo de este artículo es explicar estas diferencias. Mientras investigaciones recientes han destacado la capacidad de movilización de los oponentes para explicar la variación, se resalta aquí el impacto de factores estructurales sobre la forma y los resul-

3 Entrevistas con residentes en Chañaral, 24 de octubre de 2014. El último gran vaciado de aguas tóxicas al río ocurrió en septiembre del año 2015, ver http://www. chilesustentable.net/el-derrame-de-concentrado-decobre-de-codelco-en-Chañaral-y-sus-contradicciones/ tados de los conflictos por la minería. En detalle, se argumenta que las diferencias entre los dos casos estudiados se deben principalmente a tres factores: el peso económico relativo de la mina, así como el origen y la propiedad de la compañía involucrada. Estos tres factores configuran las oportunidades locales de al menos dos maneras: primero, influenciando la disponibilidad de recursos, alianzas y mano de obra necesaria para la movilización continua, y segundo, restringiendo oportunidades para influenciar políticas locales.

El estudio de caso sigue una lógica cualitativa y se basa en datos recolectados durante dos estadías en Chile en abril y octubre de 2015. Los datos provienen de dos fuentes: documentos secundarios como artículos de prensa nacional y local, materiales de activistas y organizaciones sociales (folletos, revistas, etc.) así como estudios académicos y entrevistas semiestructuradas. En total se condujo 25 entrevistas con activistas, líderes de organizaciones locales, personal de ONG, abogados, personal de empresas y académicos entendidos en el tema, tanto en el valle del Huasco, en Chañaral y en la capital, Santiago de Chile. ${ }^{4}$

El artículo procede de la siguiente manera: a continuación, se da un breve resumen -a manera de contextualizar- del desarrollo de la minería en Chile y la conflictividad social asociada. La siguiente sección presentará una serie de consideraciones teóricas sobre los factores estructurales que moldean los conflictos sociales en torno a la minería. Luego de esto, los casos de Pascua Lama y Chańaral serán contrastados con respecto al nivel de movilización social, las posibilidades para alianzas nacionales y transnacionales, acceso a las instituciones públicas y las reacciones de las autoridades estatales. Las causas de estas diferencias serán identificadas al presentar primero en cada capítulo la dinámica del caso de Pascua Lama y luego contrastándola con el caso de Chañaral. La última sección resume los hallazgos del estudio, provee una discusión general sobre el rol del Estado y de las compañías en los conflictos mineros en Chile y Latinoamérica y propone algunos factores a tomar en cuenta para futuras investigaciones.

4 A pedido de los entrevistados se garantiza el anonimato. 


\section{Minería y conflictos sociales en Chile}

Aunque presente desde tiempos precoloniales, fue en el siglo XIX que la minería se convirtió en el sector más importante de la economía chilena. Desde 1950 el cobre es el producto de mayor relevancia económica del país y Chile es el principal productor a nivel mundial. ${ }^{5}$ La minería, y particularmente la minería de cobre, es la base de las exportaciones nacionales y representa un porcentaje importante del PIB (real) nacional (Guajardo Beltrán, 2012). ${ }^{6}$

Hasta mediados del siglo XX la minería de cobre fue controlada por empresas privadas extranjeras (sobre todo de Estados Unidos). Esto generó un gran descontento social y político que derivó en los años sesenta en un proceso de "chilenización del cobre" que paulatinamente aumentó el control estatal sobre el sector cuprífero (Nazer, 2004). Bajo el gobierno socialista de Salvador Allende, este proceso culminó en la nacionalización del cobre, "el sueldo de Chile", en 1971. Cinco años después, en 1976, nació la empresa estatal Corporación Nacional del Cobre Codelco que controla desde entonces la producción cuprífera en el país y figura como la compañía estatal de cobre más grande del mundo (Vergara, 2011).

La dictadura de Augusto Pinochet (1973-1990) introdujo uno de los modelos neoliberales más agudos de toda la región. En el sector minero se estableció la privatización de los yacimientos de minerales (a través de "concesiones plenas" que el Estado otorga y fiscaliza) y se crearon condiciones muy favorables, incluyendo generosos incentivos tributarios para las empresas mineras (entre otras, a través del Código Minero, Nem Singh, 2010). A consecuencia, la participación privada en el sector minero creció

5 Además cuenta con importantes reservas de plata, oro, molibdeno y litio.

6 Debido a un menor grado de diversificación económica durante los ańos cincuenta y sesenta, el porcentaje de cobre asciende del 65 hasta 75\% de todas las exportaciones. En la década de los noventa el cobre proveía alrededor de un 35\% de todas las exportaciones y durante el súper ciclo aumentaba a un 50\% (Arellano, 2012a).

7 Se atribuye al presidente Allende esta expresión que se utiliza hasta hoy. de forma exponencial así como se multiplicaron las iniciativas para desarrollar actividades mineras en el país. De hecho, con un total de un $18 \%$, Chile fue el mayor recipiente de inversiones privadas en el sector minero de toda la región entre 1990 y 2001 (Bridge, 2004).

Por su importancia estratégica y su particular conexión con el Ejército chileno, ${ }^{8}$ Codelco no fue objeto de los afanes privatizadores de la dictadura: se mantuvo en propiedad pública. Hasta hoy la empresa del cobre juega un rol central en la financiación del presupuesto nacional a través de los impuestos recaudados, un porcentaje que ha crecido constantemente desde 1990, particularmente desde que el precio global del cobre incrementó su nivel de prosperidad en el año 2004 (Arellano, 2012a; Guajardo Beltrán, 2012; Lagos, 2013). Aunque el precio del cobre se encuentra en descenso desde 2013 (lo que provocó una crisis y grandes preocupaciones en Codelco y en el Gobierno de Chile), el perfil de la estatal de cobre en la economía chilena sigue siendo de importancia (Lagos, 2013). En todo tiempo Codelco ha sido visto como la "palanca del desarrollo de Chile” (Arellano, 2012b).

Con el aumento de las inversiones y el número de megaproyectos mineros, se multiplicaron también los conflictos sociales por la minería. En el presente, Chile se encuentra dentro de las naciones con una de las tasas más altas en cuanto a conflictos sociales relacionados con minería en Latinoamérica. ${ }^{9}$ Particularmente en el norte del país, una zona desértica donde se encuentran las minas de Chuquicamata (la explotación a tajo abierto más grande

8 Una particularidad chilena vincula al Ejército nacional con la producción del cobre: desde 1967 el Ejército de Chile recibe el $10 \%$ de los ingresos del cobre a través de la Ley reservada del cobre. Hasta hoy, una fuerte ala nacionalista dentro del Ejército defiende vigorosamente a Codelco como una compañía estratégica nacional (Guajardo Beltrán, 2012). De hecho, debido a su importancia fiscal la empresa constituyó una organización estratégica tanto para el gobierno militar bajo Pinochet que enfrentó muchas presiones internacionales como para los gobiernos democráticos después de 1990, que enfrentaron presiones sociales internas.

9 Siguiendo los registros del Observatorio de Conflictos Mineros en América Latina (OCMAL), ver: www.conflictosmineros.net. 
del mundo) y Radomiro Tomic, los daños ambientales y sociales son fatales (Prieto, 2014; Göbel, 2014; Castillo, 2016). En particular, la escasez de agua provocada por el uso indiscriminado que del recurso hídrico hace la minería y la contaminación del agua superficial y de las napas subterráneas ponen a los pobladores en una situación existencial muy seria.

Como en otras partes, los conflictos en torno a la minería en Chile son conflictos asimétricos. En ellos se confrontan actores locales que en su mayoría disponen de pocos recursos con autoridades estatales y megaempresas tanto nacionales como transnacionales (Romero et al., 2009; Molina, 2012; Castillo, 2016). En muchos casos, los impactos negativos de la minería se hacen presentes en comunidades y grupos que se autodefinen como indígenas y se entrelazan y refuerzan ahí con una larga tradición de exclusión y pobreza (Yánéz y Molina, 2008, 2011; Castillo, 2016). De hecho, varios estudios revelan que la resistencia a la minería intensificó y hasta incentivó los procesos de autodefinición indígena en la región (Prieto, 2014; Castillo, 2016).

\section{Contexto local, origen de las compañías y naturaleza de la propiedad}

La literatura sobre movimientos y disputas sociales, incluidos estudios sobre conflictos antimineros, tiende a acentuar las capacidades diferenciales de movilización (en el sentido de agencia con respecto a la movilización de recursos, fomento de la organización y construcción de un marco discursivo convincente) de los actores locales como el factor más importante para producir variaciones en los niveles de disputa. Sin embargo, los recursos materiales y simbólicos necesarios para iniciar una movilización no están homogéneamente disponibles, independiente del terreno en donde tiene lugar la disputa y de quienes son los actores involucrados. Más bien, ellos dependen de condiciones estructurales externas que puedan limitar o reforzar a los oponentes. ${ }^{10}$ En esta línea, Bebbington et al. (2008),

10 Para una discusión general de este argumento, ver comparando movilizaciones sociales -exitosas y fracasadas- en contra de dos proyectos mineros en Perú (Yanacocha) y Ecuador (Cotacachi) respectivamente, identifican la relativa importancia de la industria extractiva dentro de la economía nacional y territorial para determinar la fuerza de los movimientos y la posición del Estado frente a la oposición. Cuando parte de la población local percibe el proyecto como beneficioso (porque provee o proveerá ingresos y puestos laborales), la capacidad general de movilización disminuye (Arellano Yanguas, 2011). Además, cuando la industria es importante, el Estado y otras fuerzas políticas y sociales tienden a reprimir o deslegitimar aquellos movimientos (Bebbington et al., 2008). ${ }^{11}$ McAdam y Schaffer (2012), con el fin de evaluar el grado de propensión de las comunidades a oponerse a proyectos energéticos en los Estados Unidos, introdujeron el concepto de 'contexto local' (community context) que combina supuestos económicos y culturales. Este término se refiere a las variables que están fuertemente dando forma a las interpretaciones subjetivas del proyecto y en consecuencia, afectan la percepción de oportunidades y restricciones en las luchas locales (McAdam y Schaffer, 2012). Los autores teorizan que la dependencia de un lugar de la industria, así como la pobreza económica, son poderosos inhibidores de la oposición local. En el caso de los 'pueblos industriales' que dependen económicamente de industrias específicas por mucho tiempo, los habitantes locales se habitúan a los riesgos asociados a estas industrias. Como consecuencia, la mayor parte de los esfuerzos de movilización en estos casos caen en saco roto, aun cuando la contaminación amenace severamente la salud pública y el bienestar de la comunidad (ver también

McAdam y Schaffer (2012). La literatura discute las condiciones estructurales de las contiendas políticas bajo el concepto de la Estructura de Oportunidades Políticas (EOP), la cual hace referencia al sistema político institucional que configura con sus características específicas las disputas (Kriesi, 2004). Para este estudio el concepto no aplica, ya que no logra explicar la variación dentro del mismo contexto institucional.

11 Aparte del Estado, también las compañías pueden influenciar activamente los procesos de movilización: pueden fraccionar movimientos al acomodar recursos selectivamente y comprando a ciertos grupos. O también pueden presionar a los organismos estatales para alcanzar el apoyo a sus proyectos. 
Gaventa, 1980). La misma lógica opera a la luz de la pobreza económica: los habitantes locales pueden percibir el proyecto como beneficioso, y como consecuencia, no movilizarse en su contra, a pesar de las consecuencias negativas en el medio ambiente $o$ de otro tipo. Por otro lado, la existencia de alternativas económicas, así como la experiencia previa de luchas políticas, tienden a incentivar la emergencia de oposición (Arellano Yanguas, 2011; McAdam y Schaffer, 2012; Haslam y Tanimoune, 2016).

Si bien el concepto de 'contextos locales' es adecuado para explicar la emergencia y niveles generales de las disputas, no puede dar cuenta de la variación en las otras dimensiones: la escala de la movilización (local-nacional-transnacional) y las reacciones diferenciales por parte de las autoridades estatales. Se argumenta aquí que el origen y la naturaleza de la propiedad de la compañía involucrada explican estas diferencias (ver también Haslam y Tanimoune, 2016). El origen de la empresa provee oportunidades para las disputas locales a través del siguiente mecanismo: las empresas nacionales (públicas o privadas) plantean severas dificultades para producir un cambio de escala (Tarrow y Tilly, 2015) desde lo local hacia un nivel global, y la generación de lo que se conoce como 'efecto boomerang' (Keck y Sikkink, 1998). Investigaciones han demostrado que hay mayor probabilidad de que actores transnacionales apoyen causas locales si éstas se vinculan con discursos que conecten con públicos nacionales e internacionales externos (a saber, públicos en el Norte global en donde están ubicadas las grandes ONG y de donde obtienen sus recursos) (Pratt, 2012; Fløysand y Haarstad, 2007; Widener, 2007). La presencia de una compañía transnacional proveniente de Europa o Norteamérica provee este tipo de conexión. De hecho, en los últimos 20 años ha habido una emergencia de redes globales gestionadas desde el Norte. Éstas monitorean las actividades de las grandes corporaciones a nivel mundial y apoyan a los grupos locales afectados por las conductas corporativas abusivas, particularmente en el caso de la minería y la energía. ${ }^{12}$ Las implicancias para aquellas localidades dispuestas a luchar proyectos extractivos son grandes: las compañías

12 Por ejemplo 'Mining Watch' (https: $\mid \backslash$ miningwatch.ca) u 'Oil Watch' (www.oilwatch.org). transnacionales permiten estrategias transnacionales. Los habitantes locales pueden vincularse a redes transnacionales y participar en campañas internacionales. Esto, a su vez, aumenta los recursos materiales y simbólicos necesarios localmente, con el fin de sostener un conflicto en el tiempo. Más aún, provee de visibilidad global al conflicto y, como un boomerang, puede hacer presión sobre los gobiernos extranjeros que pueden, por su parte, influenciar a los gobiernos de los países en donde se realizan los proyectos (o donde supuestamente fueran a ser realizados). El activismo transnacional puede, además, impactar las evaluaciones de costos y beneficios para los accionistas de las compañías, quienes por su parte, podrían presionar a la corporación para modificar o detener las inversiones.

Contrario a esto, las empresas nacionales excluyen las alianzas transnacionales: dado que sus acciones no pueden ser fácilmente vinculadas a las experiencias en otros lugares del planeta, las compañías nacionales en Latinoamérica son menos atractivas para las redes y campañas transnacionales. Como resultado de esto, los actores locales involucrados en las disputas con estas empresas carecen de un instrumento suficientemente poderoso para presionar a los actores estatales y corporativos. De esta forma, el origen de la compañía limita tajantemente el acceso a los ámbitos globales.

La propiedad pública ejerce sus propios efectos sobre el nivel de la disputa local y, en particular, influye fuertemente sobre la reacción de las autoridades activando dos mecanismos: primero, debido a la importancia para el presupuesto y las políticas públicas, los gobiernos consideran a las compañías públicas del sector extractivo como empresas estratégicas. Como consecuencia, tienden a protegerlas en contra de demandas que tengan relación con conductas dañinas o abusivas, o medidas que puedan reducir sus ganancias (como programas de reparación del medio ambiente o multas por transgredir regulaciones). Además, existen muchas veces incentivos individuales hacia políticos para proteger a las empresas públicas; por ejemplo pueden estar involucrados directamente en las compañías como accionistas. También existe la común asignación neopatrimonial de empleos en organismos y compañías públicos a personas políticamente lea- 
les (clientelismo) (Bechle, 2010). Ciertamente este mecanismo no sucede exclusivamente en Latinoamérica: las compañías dependientes del Estado gozan de un estatus especial en todo el mundo y son constantemente acusadas de operar con poco monitoreo y bajo nivel en el control de atribuciones y responsabilidades (Revenue Watch Institute, 2013). Adicionalmente, la protección del Estado para las compañías públicas reduce la disponibilidad de otros recursos necesarios para establecer y sostener a la oposición, como por ejemplo, alianzas nacionales. Como la protección del Estado es percibida como un escudo poderoso en contra de las demandas externas, las personas tienden a desistir de la resistencia a proyectos operados por compañías públicas, pues se teme a las posibles sanciones, y a la vez, porque se percibe como abortivo.

El segundo mecanismo es de naturaleza cultural: en Sudamérica particularmente, las compañías de propiedad estatal son percibidas como fuentes legítimas de riqueza y orgullo nacional (Perrault y Valdivia, 2010). En muchos casos, el imaginario público las tiene como ejemplos vivos de una revolución nacional exitosa y de garantía de la soberanía nacional. De hecho, recobrar compañías privadas que antes fueron públicas fue una de las demandas centrales en las recientes olas de protestas antineoliberalismo, por ejemplo, en Bolivia y Ecuador (Silva, 2009). Mientras, en Venezuela y en Chile las empresas de petróleo y cobre, respectivamente, nunca han sido privatizadas. Por este motivo, es más difícil movilizar oposición en contra de la explotación 'nacional' de los recursos que movilizar resistencia en contra de actores privados o extranjeros.

\section{Oportunidades desiguales en Pascua Lama y Chañaral}

\section{Diferencias en los grados de conflicto}

En el 2001 la multinacional canadiense Barrick Gold Corp. anunció su intención de iniciar operaciones en el área de Pascua Lama. En consecuencia, varios grupos locales, incluyendo organizaciones indígenas, grupos de pequeños agricultores y agroindustriales, asociaciones religiosas y grupos urbanos movilizaron una campaña con altos niveles de participación en contra de la compañía. Emergió un frente de protesta fuerte y unificado en la región completa. Además, se formaron nuevas organizaciones para continuar con las tareas de movilización y coordinar las protestas (La Coordinadora de Defensa Alto del Carmen y el Comité de Defensa Vallenar).

El alto nivel de participación fue resultado de la estructura económica de la región. El valle del Huasco es un valle fértil, en donde parte considerable de sus habitantes se dedica a las actividades agrícolas (uva de vid y frutas). La minería en las partes más altas de la región extrae su fuerza laboral, principalmente de la capital provincial, Vallenar. Las partes restantes no dependen del sector minero. Consecuentemente, desde el comienzo Pascua Lama fue ampliamente percibido como una amenaza al suministro de agua, vital para la actividad agrícola. Productores de mayor y menor escala, es decir, tanto actores poderosos como los más débiles económicamente, comenzaron a resistir el proyecto constituyéndose en un frente de oposición masivo. Adicionalmente, la campaña en contra de Barrick se benefició de una serie de errores cometidos por la empresa. Particularmente el hecho de que no llegaron a término con su promesa de generar trabajos e ingresos (y convertir la región en una "región de compañía") y muy por el contrario, despidieron a 200 trabajadores locales. Esto encendió la indignación pública (Observatorio Latinoamericano de Conflictos Ambientales, 2012). Del mismo modo, cuando los agricultores locales revelaron los daños a los glaciares ubicados en el área concesionada, la empresa anunció que removería las masas de hielo a otro lugar, señalando que contaba con experiencia adquirida en otros lugares del mundo para realizar tal operación. Esta afirmación resultó ser falsa, lo que estimuló aún más la indignación local. ${ }^{13} \mathrm{La}$ polución de los ríos y los disturbios por el ruido de los camiones de carga pasando por las pequeñas aldeas tuvieron el mismo efecto.

Barrick reaccionó a la creciente oposición lanzando un programa social con el objetivo de cambiar

13 Ver El Chañarcillo, 8 de abril 2005; Entrevistas con activistas en el valle del Huasco y en Vallenar, 26 de octubre 2015 . 
la percepción pública sobre la minería en general y la compañía en particular (Urquidi, 2010). En este marco la empresa canalizó una impresionante cantidad de recursos financieros al gobierno regional y local, que fueron invertidos en proyectos sociales, educacionales y productivos (becas para estudiantes, apoyo financiero y técnico a clínicas, escuelas, universidades, etc.) (Schorr, 2015). Al mismo tiempo se llevó a cabo una campaña publicitaria en periódicos locales y estaciones de radio. ${ }^{14} \mathrm{La}$ empresa también proporcionó fondos y proyectos específicos a grupos sociales e individuos considerados estratégicos para ganar la llamada 'licencia social'.

Las medidas fueron temporalmente exitosas y la oposición se diluyó (Urquidi, 2010). El proyecto finalmente comenzó en el ańo 2009, apoyado por autoridades locales y regionales. Sin embargo la resistencia local no cesó totalmente. Una masa crítica de activistas continuó movilizando e inició procedimientos legales (ver abajo). Las protestas continuas, las sanciones legales y una serie de problemas técnicos provocaron un alza significativa en los costos de la empresa, lo que desencadenó presión por parte de los accionistas. Como resultado, Barrick detuvo operaciones en el año 2012 y luego suspendió sus actividades hasta el año 2017. ${ }^{15}$

En Chañaral, las movilizaciones en contra de la minería y la polución derivada de ella comenzaron a finales de la década de los setenta. Varios grupos sociales (sindicatos, organizaciones vecinales, clubes deportivos, etc.) se unieron para formar un comité ciudadano (comité cívico) para coordinar acciones en contra de la compañía (Vergara, 2011). Solicitaron a Codelco detener la contaminación y reparar los daños causados. Sin embargo, comparado con Pascua Lama, las protestas públicas y el apoyo a los activistas fueron bajos, y luego del fallo

14 Entrevista con activistas en el valle del Huasco y en Vallenar, 26 de octubre 2015.

15 Ver http://www.economiaynegocios.cl/noticias/noticias.asp?id=179772. Debido a varias demandas e incumplimientos ambientales por parte de Barrick, la Superintendencia de Medio Ambiente (SMA) de Chile sancionó en enero 2018 con la clausura definitiva del proyecto minero Pascua Lama (ver http://www.sma. gob.cl/index.php/noticias/comunicados/911-sma-sanciona-a-pascua-lama-2018) del Tribunal Constitucional en 1988 que mandó a Codelco construir un dique de contención la participación declinó aún más. Hasta hoy, un grupo de activistas de la ONG Chadenatur (fundada en 2001) intenta movilizar apoyo y continúa la disputa contra Codelco. ${ }^{16}$ Pero siguen enfrentando mayores dificultades movilizando a los locales en contra de la compañía y la mina por varias razones:

Primero, tal como en la zona del Huasco, la estructura económica regional afecta el nivel de disputa en contra de la mina; en este caso la restringe. De hecho, Chañaral es una especie de clásico 'pueblo de compañía' (company towns). La minería, en particular Codelco, es el empleador más importante en la ciudad (de hecho, de la región en general; ver Vergara, 2011, y Guajardo Beltrán, 2012). Datos proporcionados por el Congreso Nacional señalan que alrededor de dos tercios de los habitantes trabajan en minería y actividades relacionadas a ella. ${ }^{17}$ La gente teme perder la única fuente de ingreso confiable si los grupos de activistas tuvieran éxito, ya que ellos "viven de la mina". ${ }^{18}$

Además, la naturaleza de la propiedad de la compañía limita la movilización en Chañaral, así como en cualquier otro lugar de Chile. Codelco es visto (especialmente por la gente mayor) como el pilar económico más importante del país, y como proveedor del "salario de Chile". Más aún, hay una fuerte memoria colectiva de Codelco, como resultado de una intensa lucha social que culminó en la apropiación de los recursos naturales a través de la nacionalización (Guajardo Beltrán, 2012, ver sección anterior). Para muchos chilenos Codelco es un símbolo de soberanía nacional y una garantía de sobrevivencia y bienestar nacional. De manera ilustrativa, en una encuesta del año 2009, el 51\% de los encuestados consideraron a Codelco como la compañía más importante del país. ${ }^{19}$ Movilizarse en contra de tal emblema y símbolo nacional es una tarea difícil de alcanzar, a nivel local y nacional.

16 Para más información acerca de Chadenatur, ver: www. desastre-ecologico.cl

17 Ver http://reportescomunales.bcn.cl

18 Entrevista con activistas, Chañaral, 24 de octubre 2015.

19 https://www.codelco.com/prontus_codelco/site/artic/20110803/asocfile/20110803121111/codelco_informa_04.pdf. 
Otro impedimento general para la movilización deriva del hecho de que la polución existe desde un período muy largo de tiempo. Los activistas explican que las personas en Chañaral están acostumbradas a la contaminación causada por la mina, por lo tanto no la conciben como algo creado por humanos $y$, en consecuencia, como algo que debiese ser abordado por la política. De hecho, han crecido ya dos generaciones en el pueblo de Chañaral sin haber conocido otro paisaje del lugar aparte del contaminado. La contaminación se ha transformado en una parte intrínseca en sus vidas. Como resultado, la participación en protestas en contra de la mina o la compañía está fuera del espectro para muchas personas. Como bien lo expresó un activista: "Uno está tentado a decir que el problema más grande de Chañaral son las mismas personas de Chañaral" ${ }^{20}$

Por último, como hizo Barrick en el caso de Pascua Lama, Codelco ejecutó una gran campaña social desde 1990 en adelante, siendo capaces de influenciar la opinión pública con gestos simbólicos, como la construcción del faro o el financiamiento de una orquesta (Cortés, 2014). Adicionalmente, estableció fuertes alianzas con actores locales significativos (mayormente grupos vecinales y de negocios). En 2001, la compañía firmó una alianza estratégica con la municipalidad llamada 'Buen Vecino'. Ofrecieron actividades recreacionales, tales como caminatas públicas los domingos en la tarde, curiosamente llamadas 'caminatas por la salud'21, además de aportar con fondos para apoyar programas locales de productividad, educación (programa de becas para estudiantes universitarios) y de capacitación. ${ }^{22}$ En contraste con Pascua Lama, Codelco se benefició del apoyo incondicional de la Municipalidad de Chañaral, que proveía el acceso a su densa red de clientes (ver abajo). Combinado a los otros factores, la alocación estratégica de recursos a ciertos grupos sociales apoyada por la municipalidad ayudó a mantener bajos los niveles de movilización pública.

20 Entrevista con activistas, Chañaral, 24 de octubre 2015.

21 http://www.soychile.cl/Copiapo/Socie$\mathrm{dad} / 2011 / 12 / 13 / 57835 /$ Las-caminatas-familiaresBuen-Vecino-fueron-un--exito-en-Chañaral-y-Diegode-Almagro.aspx

22 https://www.codelco.com/buen-vecino-Chañaral/ prontus_codelco/2011-08-09/112734.html

\section{Desigualdad en las Escalas de Conflicto}

Poco después de estallar el conflicto, los activistas en el valle del Huasco establecieron contacto con y fueron contactados por ONG de la capital, Santiago (Oceana, Instituto de Ecología Política, Chile Sustentable, Greenpeace y el Observatorio Latinoamericano de Conflictos Ambientales (OLCA (Rivera, 2011). El OLCA fue particularmente importante, multiplicando el apoyo y la solidaridad con los activistas locales (OLCA, 2011; Salinas, 2007). Como miembro de varias redes nacionales e internacionales preocupadas por temas ambientales, actuó como un agente que vinculó las luchas locales del valle con organizaciones nacionales e internacionales, así como también a redes transnacionales. A nivel nacional, OLCA trabajó para dar visibilidad al caso organizando protestas frente al Congreso y la oficina de Barrick en Santiago, también realizando discusiones públicas sobre minería y sus impactos en general, enfocándose en el conflicto de Pascua Lama en particular. Esta mayor visibilidad atrajo a más ONG y derivó en que estudiantes formaron grupos de solidaridad en Santiago y otras ciudades.

A nivel transnacional, OLCA proporcionó contacto con redes globales. Las condiciones fueron más bien propicias. Barrick Gold Corp. de Canadá es una de las mineras más grandes del mundo, que además posee un récord mundial en conductas dañinas para el medio ambiente. Esto ha llevado a la formación de diversos grupos de defensa transnacionales, muchos de ellos en Canadá mismo. De hecho, aunque Canadá es una sólida nación minera (como Chile), es también hogar de un sinnúmero de Organizaciones No Gubernamentales y grupos de ciudadanos que resisten a la minería y a las compañías mineras (especialmente las canadienses). Estos grupos monitorean y se movilizan en contra de las compañías cuando se reportan conflictos (Rivera, 2011). Específicamente en contra de Barrick se creó "Protestbarrick.net", un portal de internet que grupos de activistas ocupan para coordinar acciones en contra de la corporación multinacional en todo el mundo. ${ }^{23}$ La agrupación "Protest Barrick", por su parte, es miembro de la "Red de Solidaridad frente

23 Ver http://protestbarrick.net/ 
a la Injusticia Minera" (Mining Injustice Solidarity Network, MISN), que apoya a las comunidades afectadas por la minería canadiense en todo el mundo. ${ }^{24}$ Por lo tanto, la presencia de Barrick permitió a activistas locales acceder a redes transnacionales sin costos significativos. El caso de Pascua Lama se incluyó en la larga lista de comunidades afectadas por Barrick y se difundió globalmente un llamado a la solidaridad. Los grupos de defensa también desplegaron una serie de actividades, como por ejemplo, en 2007 "Protest Barrick" realizó una campaña internacional y coordinó protestas en contra de la empresa en el mundo entero. En otra ocasión, grupos indígenas de todo el mundo fueron invitados a Canadá con el fin de hablar en la reunión anual de accionistas de Barrick en Toronto. Adicionalmente, se formaron grupos canadienses de solidaridad específicos (como el 'Grupo No A Pascua Lama' en Montreal).

Otro actor transnacional importante en el conflicto sobre Pascua Lama fue Greenpeace. A través de su capítulo chileno, la ONG incorporó el conflicto a su campaña global de cambio climático y protección de glaciares (Rivera, 2011). Al hacer esto contribuyó significativamente al aumento de visibilidad del conflicto a nivel mundial y en el mismo Chile. Además de difundir la información del caso, Greenpeace proporcionó material de apoyo. Por ejemplo, publicando un boletín para inversionistas que contenía información técnica detallada de violaciones legales, daños medioambientales y demandas pendientes en contra de Pascua Lama. ${ }^{25}$ En 2015 Greenpeace Chile abrió otro frente para Barrick, filtrando información acerca de la existencia de vertederos de desechos en los glaciares dentro del área concesionada de Pascua Lama. ${ }^{26}$

24 Ver http://mininginjustice.org/. Hay varias otras ONG y redes basadas en Canadá, ver https://miningwatch.ca

25 Entrevistas con activistas en el valle del Huasco y Alto del Carmen, 21 de octubre 2015. El informe de Greenpeace para los accionistas de Barrick se encuentra en: http://www.greenpeace.org/argentina/Global/argentina/report/2013/cambio_climatico/accionistas-completo-revisado-gs-ss.pdf

26 http://radio.uchile.cl/2015/10/18/greenpeace-denuncia-basurales-en-glaciares-intervenidos-por-proyectopascua-lama
La estrategia multiescalar para resistir a Pascua Lama combinó instrumentos extrainstitucionales (como marchas y bloqueos), institucionales (audiencias en el Parlamento, investigaciones del Congreso, quejas ante la autoridad ambiental) y legales. En cuanto a esta última, los grupos locales recibieron el apoyo y servicios por parte de diversos abogados. ${ }^{27}$ Este apoyo fue decisivo para el curso del conflicto: fueron presentadas muchas demandas a los tribunales chilenos (ver próxima sección). Adicionalmente, una particularidad regional facilitó la apelación a la ley internacional: el valle del Huasco es hogar del grupo indígena de los diaguitas, formalmente acreditado en 2006 por el Estado chileno. Tres años después, Chile ratificó el Convenio 169 de la OIT que reconoce derechos específicos a la autodeterminación de los grupos indígenas. Esto abrió la oportunidad de reclamar la participación de la comunidad diaguita en las decisiones sobre Pascua Lama, a través de un proceso de consulta previa dispuesto por el convenio. Asesorado por abogados expertos, la Comunidad Agrícola Diaguita de los Huascoaltinos primero apeló sin éxito a una corte chilena, para luego presentar una demanda contra el Estado chileno a la Corte Interamericana de Derechos Humanos por no haber sido consultados apropiadamente (Molina, 2013). La sentencia está pendiente hasta la actualidad.

El caso de Pascua Lama constituye un marcado contraste con lo que ocurrió en Chañaral, en donde los grupos locales no fueron capaces de llevar su causa más allá del nivel local y generar apoyo nacional o transnacional. Aunque activistas de Chadenatur mantienen un contacto cercano con las ONG nacionales -como Chile Sustentable y OLCA-, y participan en diversas iniciativas medioambientales nacionales, no han alcanzado la participación activa de ninguno de ellos. Según se informa, se ha solicitado asistencia a OLCA, y ellos se han mostrado muy interesados, sin embargo se han sentido incómodos de involucrarse en un conflicto con niveles tan bajos de apoyo público a la causa. ${ }^{28}$ Esto, a su vez, es resultado de la estructura económica

27 Entrevistas con activistas en el valle del Huasco y Alto del Carmen, 21 de octubre 2015.

28 Entrevista con activistas en Chañaral, Chañaral, 24 de octubre 2015. 
dominante y de la naturaleza de la propiedad de la empresa. Tampoco tuvieron éxito en su acercamiento a Greenpeace. Se dice que ellos se rehusaron porque el caso no calza dentro de su campo estratégico de actividades: "No tenemos glaciares", como explicó un activista local.

Del mismo modo, hasta hoy ningún abogado ha aceptado llevar el caso contra Codelco a la Corte. Esta ausencia, en un país con una fuerte tendencia a judicializar a sus oponentes en situaciones conflictivas, es particularmente extraña. Nuevamente, los activistas de Chańaral reportan que los abogados consideran su caso 'desesperanzador', porque perciben que nadie va a ganar contra la poderosa empresa pública. ${ }^{29}$ En lo referente a las redes transnacionales, los registros de activistas en Chañaral son, también, negativos. Como en el caso de ONG nacionales, intentaron conectarse a las redes de defensa transnacional, pero encontraron muy difícil conseguir apoyo. Los locales suponen que el origen de la empresa explica su fracaso. El caso de Codelco - una compañía chilena nacional- no se conecta con el universo discursivo y estratégico de la mayoría de las redes de defensa transnacional y, en consecuencia, 'no es atractivo' ${ }^{30}$ para campańas globales.

\section{Desigualdad en accesos institucionales}

Activistas en Pascua Lama no solo lograron fuerte apoyo extrainstitucional, sino que también se conectaron a instituciones políticas nacionales. Esto fue posible principalmente por la coyuntura política en Chile: las elecciones generales del año 2013 introdujeron a cuatro jóvenes exlíderes del movimiento estudiantil del año 2011 al Congreso. Aunque fueron electos bajo diferentes afiliaciones partidarias, formaron un grupo llamado la 'bancada juvenil' que traería al Congreso un rango de nuevas propuestas de reforma. Los activistas del valle del Huasco contactaron a este grupo y obtuvieron su apoyo. ${ }^{31}$ Como consecuencia, presionaron para el establecimiento de un comité de investigación de la Cámara de Diputados sobre supuestas

29 Ídem.

30 Ídem.

31 Entrevista con activistas locales en el valle del Huasco, Alto del Carmen, 22 de octubre 2015. irregularidades durante el proceso de concesión y el comportamiento medioambiental de Barrick. ${ }^{32}$ Las audiencias y las visitas a terreno ocurrieron entre septiembre de 2013 y enero de $2014 .{ }^{33}$ La comisión concluyó sin confirmar los cargos de los activistas en contra de la empresa. Pero, su establecimiento y trabajo ampliamente reportado en los medios nacionales ayudó al movimiento anti Pascua Lama a ganar visibilidad. Esto fortaleció a los activistas y a los grupos de solidaridad. ${ }^{34}$

Los activistas del valle del Huasco también obtuvieron apoyo de autoridades subnacionales. Desde el comienzo del conflicto, y motivados por las mismas razones que los activistas, algunos políticos locales (miembros del consejo local, etc.) se opusieron al proyecto y apoyaron acciones extra e intrainstitucionales en su contra (Salinas, 2007).

Por el contrario, los activistas en Chañaral intentaron en contadas ocasiones contactar a diputados nacionales que estuviesen dispuestos a ejercer presión para el establecimiento de una comisión oficial de investigación de la polución y las responsabilidades asociadas. No tuvieron éxito. Hasta ahora, no existe ningún estudio oficial que confirme la contaminación en Chańaral e identifique a Codelco como el agente responsable. Los diversos estudios privados realizados por prestigiosos científicos no han sido reconocidos por autoridades locales o nacionales (Cortés, 2014). A comienzos de los noventa el comité cívico consiguió el apoyo del Congreso para aprobar una ley que establecería el pago de compensaciones con recursos públicos que debían ser invertidos en proyectos infraestructurales en la región. Al concernir recursos nacionales, esta ley necesitaba la aprobación presidencial y el presidente Patricio Aylwin se negó a aprobarla (Cortés, 2014). Solo recientemente, los activistas dieron con una delegada del Partido Comunista que abrió al menos parcialmente las puertas al Congreso: miembros de

32 “Comisión Especial Investigadora acerca de la situación ambiental en que se encuentran las comunas del valle del Huasco, región de Atacama, por los trabajos de instalación del Proyecto Pascua Lama"

33 https://www.camara.cl/trabajamos/comision_portada. aspx?prmID $=737$

34 Entrevista con activistas locales en el valle del Huasco, Alto del Carmen, 22 de octubre 2015. 
Chadenatur fueron a Santiago a exponer su caso a la Comisión del Medio Ambiente y Recursos Naturales de la Cámara de Diputados. Hasta ahora, no ha habido consecuencias. ${ }^{35}$

\section{Desigualdad en las respuestas por parte de las autoridades estatales}

Desde el inicio del proyecto varios gobiernos chilenos, así como autoridades locales y regionales, han apoyado fuertemente el proyecto de Pascua Lama, por las ganancias directas e indirectas esperadas (ingresos fiscales, empleos, incentivo a los negocios locales, etc.) (Salinas, 2007). A pesar de este respaldo, Barrick tuvo que enfrentar cargos de la Superintendencia del Medio Ambiente y del Tribunal Ambiental que habían admitido los alegatos en contra de la empresa y que finalmente sancionaron en favor de la oposición local. De hecho, la corte y los organismos ambientales fueron claves para debilitar a la empresa en el conflicto, alzando sus costos. Por ejemplo, luego de haber realizado la primera evaluación medioambiental, la Comisión Nacional de Medio Ambiente, Conama, solicitó a Barrick presentar un plan de manejo de glaciares y así retrasaron la iniciación del proyecto. Del mismo modo, la Superintendencia del Medio Ambiente (organismo estatal encargado de monitorear las inversiones con implicaciones ambientales) abrió varios procesos sancionadores en contra de Barrick. En el año 2013 la compañía recibió una multa de 8 mil millones de pesos chilenos por haber transgredido regulaciones ambientales tal como se define en el plan ambiental del proyecto (resolución de calificación ambiental). ${ }^{36}$ En 2018, la Superintendencia de Medio Ambiente finalmente mandó clausurar definitivamente el proyecto Pascua Lama. Asimismo, la Comisión de Investigación de la Cámara de Diputados que investigó las infracciones ambientales de Barrick Gold en el caso de Pascua criticó en su informe fuertemente a las instituciones públicas involucradas en la concesión y en el monitoreo del proyecto y apoyó varias de las demandas

35 Entrevista con activistas locales, Chañaral, 24 de octubre 2015 .

$36 \mathrm{http}: / / \mathrm{www}$.lanacion.cl/sancionan-con-8-000-millones-a-barrick-gold-por-pascua-lama/noticias/2013-05-24/133354.html de las comunidades del valle de Huasco. ${ }^{37}$ Además, recomendó una serie de reformas legislativas para reforzar, entre otros, la gestión ambiental. ${ }^{38}$

Mientras que en Pascual Lama el gobierno, a pesar de apoyar el proyecto, no intervino directamente, y ciertos organismos estatales se organizaron para lidiar con sus déficits medioambientales, en Chañaral todos apoyaron a la empresa. Las autoridades nacionales, regionales y locales han negado incesantemente la existencia de un problema ambiental severo causado por Codelco. Hasta hoy, ni el gobierno local ni la intendencia y aún menos el gobierno nacional han hecho algo para abordar la dramática situación, a pesar de años de denuncias y acciones públicas de los habitantes locales (Cortés, 2014). Muy por el contrario, después del mandato del Tribunal Constitucional en 1988, resultado de la apertura democrática del momento, los organismos estatales junto a la empresa dirigieron una extensa campaña pública destinada a probar que las preocupaciones medioambientales no tenían base, y en consecuencia, eran inválidas (Cortés, 2014). El clímax de esta campaña se alcanzó en el año 2003 cuando el entonces presidente Ricardo Lagos hizo una visita sorpresa a Chańaral. Junto al alcalde de entonces, Héctor Volta (2000-2016), y la intendenta de la región de Atacama, Yasna Provoste, el presidente tomó un baño en las aguas de la bahía para demostrar públicamente que la contaminación no era algo de lo que debían preocuparse. Lagos fue acompañado por el gerente general de División Salvador de Codelco. La presencia de la intendenta Provoste es, también, curiosa. Después de haber ejercido varios cargos (ministra de Planificación y Cooperación en el año 2004 y de Educación en el año 2006), hoy es diputada del Distrito $\mathrm{N}^{\circ} 6$ que incluye varias municipalidades en el valle del Huasco, entre ellas Alto del Carmen y Vallenar. Desde su posición, se opone fuertemente a Pascua Lama. Otro elemento dentro de la campańa pública para apoyar a Codelco fue el establecimiento de balnearios en la bahía de Chañaral -en una de las áreas

37 Ver http://www.chilesustentable.net/comision-de-camara-de-diputados-emitio-contundente-informe-encontra-de-pascua-lama/

38 El informe se encuentra aquí: https:/www.camara.cl/ pdf.aspx?prmID=10839\&prmTIPO=INFORMECO MISION 
más contaminadas del planeta-. Al ser exigido su cierre por activistas locales, pues estos balnearios carecían de la aprobación sanitaria necesaria para operar, la Seremi (Secretaría Regional Ministerial) de Salud de Antofagasta se rehusó a hacerlo. ${ }^{39}$ Del mismo modo, los organismos ambientales han mantenido el silencio hasta el presente. En 1998, la Comisión Regional Medioambiental, Corema, publicó un estudio dirigido por el Centro de Investigación Minera y Metalúrgica que no confirmó los graves niveles de polución (Cortés, 2014). Recientemente, la ministra de Salud afirmó que Chañaral no es un lugar preocupante en términos medioambientales. ${ }^{40}$

Parece ser que los activistas se estrellan contra una gran pared al intentar que Codelco se responsabilice por la polución y sus consecuencias. Organismos estatales, en diversos niveles, han activamente apoyado a la compañía o se han quedado inertes en cuanto a los alegatos locales. De hecho, desde su creación, Codelco se ha beneficiado de una sorprendente defensa corporativa por parte de las autoridades estatales, motivada por la importancia económica para el Estado de Chile (Molina, 2012). Solo recientemente, en la inauguración de una nueva mina de Codelco, la entonces presidenta de Chile, Michelle Bachelet, enfatizó que "estamos marcando un hito histórico en la empresa de todos: estamos dando el primer paso - de los muchos que daremos- para asegurarnos de que Codelco se mantenga en la vanguardia de la minería mundial y siga impulsando el desarrollo de Chile". La nueva

39 Más adelante, el Ministerio de Salud se retractó parcialmente y solicitó a la Municipalidad de Chañaral la instalación de signos de precaución que señalaran que bañarse en la bahía constituía un riesgo para la salud. Sin embargo la municipalidad no acató y las señales nunca fueron montadas (Cortés, 2014).

40 http://www.semanario7dias.cl/ministra-de-saludasegura-que-la-contaminacion-historicamente-no-hapreocupado-a-Chańaral/. Curiosamente, en lo que se refiere a la economía local, la presencia de una cantidad impresionante de metales pesados en la bahía no se niega: desde hace un par de ańos, las autoridades locales y regionales proponen un proyecto para explotar los desechos tóxicos de la bahía (los cuales contienen cobre). Hasta ahora, no han encontrado inversionistas, aunque varias compañías se han mostrado interesadas (Cortés, 2014). operación minera, agregó, responde "a un interés puramente nacional". ${ }^{41}$ Hasta Moodys, la agencia de calificación estadounidense, reconoce el fuerte apoyo del Gobierno de Chile y la alta dependencia entre Codelco y el gobierno, como críticos para una calificación general positiva. ${ }^{42}$ Como consecuencia, los activistas de Chañaral no han podido lograr una atención a sus demandas por parte del Estado.

\section{Conclusión}

El objetivo de este artículo es resaltar las limitaciones estructurales más allá del control directo de los oponentes locales en cuanto a las oportunidades de expresión de voz e impacto político en el contexto de conflictos por la minería. A través de los dos casos en el valle del Huasco y la comunidad de Chañaral en Chile se mostró que el grado, el alcance y los resultados de las disputas sobre la minería dependen de tres factores: primero, el contexto local específico, particularmente la dependencia económica de la mina y la duración general de la operación; segundo, el origen de la empresa involucrada $y$, tercero, la naturaleza de la propiedad de la compañía. En detalle, la dependencia económica del lugar tiende a reducir el grado de movilización local y el origen nacional (no importa si público o privado) dificulta alianzas transnacionales. La propiedad pública ejerce dos efectos: por su peso simbólico y económico reduce, al igual que el contexto local, el nivel de participación. Además, restringe las reacciones políticas en contra de las empresas.

Los casos ilustran estos argumentos: el valle del Huasco no depende de la minería, sino de la agricultura. Como consecuencia, las amenazas percibidas por el proyecto minero, y los daños medioambientales producidos durante la fase exploratoria, llevaron a la movilización de una alianza multisectorial que incluyó actores económicos fuertes y débiles. Este alto nivel de resistencia local motivó la solidaridad

41 http://www.gob.cl/president-bachelet-today-we-cansay-that-the-chilean-government-is-beginning-to-repay-its-debt-to-codelco/

42 https://www.codelco.com/prontus_codelco/site/ artic/20150902/asocfile/20150902171034/moody_ codelcocreditopinion_jul2015.pdf 
nacional y a abogados a tomar el caso. Además, la presencia de la empresa transnacional canadiense, conocida globalmente por su comportamiento depredador, permitió fácilmente la conexión a redes de defensa globales y la ampliación del campo de negociación a nivel global. Más aún, la oposición local alcanzó acceso a instituciones nacionales y logró llamar a los organismos estatales a la acción.

El contraste con Chañaral no puede ser más agudo. Desde hace muchas décadas Chañaral y la región en general dependen fuertemente del sector minero, particularmente de las minas de cobre administradas hoy por Codelco. Como consecuencia, a los activistas en Chañaral les resulta difícil movilizar apoyo en contra de la mina y de la empresa para lograr una indemnización y reparación por los daños causados. Muchas veces, los locales prefieren no movilizarse en contra de su única fuente de ingresos. Más aún, debido a la cantidad de tiempo de coexistencia con la mina y la polución, muchos ni siquiera perciben la contaminación como peligrosa. El bajo nivel de apoyo a los activistas en Chañaral es también resultado del importante rol de Codelco en la economía nacional, así como su posición en el imaginario nacional chileno. La compañía está considerada como vital para la soberanía, el bienestar y los intereses nacionales. Este enorme capital simbólico y económico es lo que mantiene a las personas lejos de la oposición. Por el mismo motivo, los activistas locales no lograron el apoyo de instituciones políticas, reacias a respaldar iniciativas que pudiesen de algún modo afectar al gigante público del cobre. Adicionalmente, el hecho de que los gobiernos chilenos protejan firmemente 'su' compañía parece haber influenciado a potenciales partidarios en términos del cálculo entre los costos y beneficios de sumarse a la causa: particularmente en el caso de abogados que perciben una victoria legal en contra de la empresa pública como algo inviable. Finalmente, las autoridades estatales de diversos niveles y organismos, incluyendo los medioambientales, no han tomado partido por la población local en sus 30 años de lucha en contra de la minería. Muy por el contrario, la compañía ha sido respaldada por autoridades nacionales, regionales y locales.

Si bien este argumento se centra en las empresas, también incluye al Estado como 'dueño' de la com- pañía dentro del análisis. Desde una perspectiva más general, los casos de Pascua Lama y Chańaral resaltan el complejo rol del Estado en la disputa sobre la minería. Aparentemente el Estado chileno no se comporta como un actor equilibrado y consistente en este tipo de conflictos. Esto es un indicativo del hecho general de que dentro de la caja negra del Estado existe un sistema complejo de fuerzas con misiones e intereses parciales en oposición. Estas diferencias se hacen evidentes en algunas situaciones, como cuando los organismos medioambientales o de derechos humanos actúan en nombre de los grupos locales oponiéndose a un proyecto que el Ministerio de Economía y los gobiernos apoyan firmemente. Sin embargo, como revela el caso de Chañaral, puede haber situaciones en donde las jerarquías dentro del Estado se imponen. Un ejemplo de esto es cuando se ve afectada la compañía pública con su importancia económica.

Cierto es que la fuerza explicativa de los factores identificados como causa de las oportunidades desiguales en la disputa sobre la minería se limita a los dos casos chilenos. Sin embargo, la variación de niveles, escalas y resultados de los conflictos puede ser observada también en otros países de Latinoamérica. El enfoque en el contexto local, el origen y la propiedad de la compañía puede ayudar a entender estas diferencias en otros emplazamientos mineros, algunos más controversiales que otros, por ejemplo en Bolivia, Perú o Ecuador. Además, los tres factores por supuesto no son las únicas causas que explican diferencias en la disputa local en contra de la minería en Chile o en otras partes. Sin embargo, lo que estos factores sugieren es que con el fin de explicar la variación en la disputa sobre la minería, es necesario tomar en cuenta el contexto social, económico y cultural en el que el proyecto minero está operando. Por ejemplo, los niveles de movilización pueden depender también de la agencia y habilidades de los movilizadores. Del mismo modo, la existencia de una masa crítica de gente joven que sea ecológicamente sensible y con participación política puede marcar una diferencia (como sería el caso de Pascua Lama). Además, el apoyo transnacional puede depender de otros factores que el origen de la empresa, tales como especificidades geográficas como por ejemplo la existencia de glaciares que activaron el apoyo de Greenpeace en el 
conflicto de Pascua Lama. La existencia de grupos indígenas también marcaría diferencias, aunque la evidencia empírica parece ambigua. Los estudios de Prieto (2014) y Castillo (2016), por ejemplo, demuestran que la posibilidad de recurrir a una identidad indígena que implique una tradición cultural distinta basada en un pasado ancestral imaginado puede generar un alto grado de cohesión social. Lo que por su parte promovería una gran predisposición individual de participar en actividades en favor de la colectividad. Ambos autores mencionan también el acceso a las leyes internacionales (Convenio 169) que posibilita la autoidentificación indígena como instrumento efectivo para lograr demandas en el contexto de conflictos mineros. Otros trabajos son más críticos, por ejemplo Schilling-Vacaflor (2014), que muestra que el impacto de las consultas previas en conflictos locales por proyectos extractivistas es muy reducido. Al final de cuentas son los Estados los que implementan la jurisprudencia internacional. Por último, el origen nacional de una empresa también puede influenciar las reacciones del Estado si la gerencia o los dueños gozan de un acceso preferencial a las decisiones públicas. Estos y muchos temas más quedan abiertos para investigaciones futuras.

\section{Agradecimientos}

Quiero agradecer el apoyo en la elaboración de este artículo a mis colegas Marianne Braig (FU Berlín), Rodrigo Rodrigues-Silveira (Universidad de Salamanca) y Veronica Schild (University of Western, Ontario). También agradezco los valiosos comentarios de los evaluadores anónimos. A Frauke Berg y a Margarita Vergara quiero agradecer por la ayuda en la edición y traducción del documento.

\section{Referencias citadas}

Alimonda, H. (2011) (Coord.). La Naturaleza colonizada. Ecología politica y minería en América Latina. Buenos Aires: Clacso.

Arellano, J. P. (2012a). Copper Mining and its Impact on Chile's Development. Integration and Trade. Integración y Comercio, 35(16), 45-57.
Arellano, J. P. (2012b). El cobre como palanca del desarrollo para Chile. Estudios Públicos, 127.

Arellano Yanguas, J. (2011). Aggravating the Resource Curse: Decentralization, Mining and Conflict in Peru. Journal of Development Studies, 47(4), 617-638.

Barton, J., Bustos, B. y Prieto, M. (2015) (Coords.). Ecología Politica en Chile: naturaleza, propiedad, conocimiento y poder. Santiago: Universitaria.

Bebbington, A. (2012) (Coord.). Social Conflict, Economic Development and Extractive Industry: Evidences from South America. London: Routledge.

Bebbington, A. \& Bury, J. (2013) (Coords.). Subterranean Struggles. New Dynamics of Mining, Oil and Gas in Latin America. Austin: University of Texas Press.

Bebbington, A., Humphreys-Bebbington, D., Bury, J., Lingan, J. \& Muñoz, J. P. (2008). Mining and Social Movements: Struggles Over Livelihood and Rural Territorial Development in the Andes. World Development, 36(12), 2888-2905.

Bechle, K. (2010). Neopatrimonialism in Latin America: Prospects and Promises of a Neglected Concept, GIGA Working Papers num. 153, Hamburg: GIGA.

Bridge, G. (2004). Mapping the Bonanza: Geographies of Mining Investment in an Era of Neoliberal Reform. The Professional Geographer, 56(3), 406-421.

Burchardt, H.-J. \& Dietz, K. (2014). (Neo-) extractivism A New Challenge for Development Theory from Latin America. Third World Quarterly, 35(3), 468-486.

Castillo, M. (2016). Socio-Ecological Inequality and Water Crisis: Views of Indigenous Communities in the Alto Loa Area. Environmental Justice, 9(1), 9-14.

Cortés, M. (2014). La muerte gris de Chañaral. La vergüenza de la División Salvador de Codelco-Chile. Chañaral: Impresos Plaza.

Dietz, K. \& Engels, B. (2016) (Coords.). Contested Extractivism. Basingstoke: Palgrave Macmillan.

Fløysand, A. \& Haarstad, H. (2007). Globalization and the Power of Rescaled Narratives: A Case of Opposition to Mining in Tambogrande, Peru. Political Geography, 26, 289-308. 
Gaventa, J. (1980). Power and Powerlessness: Quiescence and Rebellion in an Appalachian Valley. Chicago: University of Illinois Press.

Göbel, B. (2014). La minería de litio en Atacama: disputas sociales alrededor de un nuevo mineral estratégico. En Göbel, B. y Ulloa, A. (Coords.). Extractivismo minero en América Latina. Territorios indigenas y campesinos y sus interdependencias transregionales. Serie Perspectivas Ambientales. Bogotá/Berlín: Universidad Nacional de Colombia / Ibero-Amerikanisches Institut, 167-193.

Göbel, B. y Ulloa, A. (2014). Extractivismo minero en América Latina. Territorios indigenas y campesinos y sus interdependencias transregionales. Serie Perspectivas Ambientales. Bogotá/Berlín: Universidad Nacional de Colombia / Ibero-Amerikanisches Institut.

Göbel, B., Góngora-Mera, M. y Ulloa, A. (2014). Desigualdades socioambientales en América Latina, Serie Perspectivas Ambientales. Bogotá/Berlín: Universidad Nacional de Colombia and Ibero-Amerikanisches Institut.

Guajardo Beltrán, J. C. (2012). Mineral Rents and Social Development in Chile. En Hujo, K. (Coord.). Mineral Rents and the Financing of Social Policy. Opportunities and Challenges. Basingstoke: Palgrave MacMillan, 185-222.

Gudynas, E. (2009). Diez tesis urgentes sobre el Nuevo Extractivismo: contextos y demandas bajo el progresismo sudamericano actual. En Acosta, A., Barandiará, A., Bebbington, A., Folchi, M., Alayza, A., Gudynas, E., Schuldt, J. Extractivismo, Politica y Sociedad, Quito: CAAP/CLAES, 187-225.

Haslam, P. A. \& Tanimoune, N. A. (2016). The Determinants of Social Conflict in the Latin American Mining Sector: New Evidence with Quantitative Data. World Development, 78, 401-419.

Karmy, J. y Salinas, B. (2009). Pascua Lama: conflicto armado a nuestras espaldas. Santiago de Chile: Quimantú.

Keck, M. \& Sikkink, K. (1998). Activists beyond Borders. Advocacy Networks in International Politics. New York: Cornell University Press.

Kriesi, H. (2004). Political Context and Opportunity. En Soule, S. et al. (Coords.). The Blackwell Companion to Social Movements. Oxford: Blackwell Publishing, 67-90.
Lagos, G. (2013). El superciclo del cobre y sus efectos en Chile. Programa de Investigación en Economía de Minerales, Universidad Católica de Chile, Santiago, Chile.

McAdam, D. \& Schaffer, H. (2012). Putting Social Movements in their Place. Explaining Opposition to Energy Projects in the United States, 2000-2005. Cambridge: Cambridge University Press.

Molina, F. (2012). Competing Rationalities in Water Conflict: Mining and the Indigenous Community in Chiu Chiu, El Loa Province, Northern Chile, Singapore Journal of Tropical Geography, 33, 93-107.

Molina, R. (2013). Diaguitas: Emergencia étnica y dialéctica de las relaciones y conflictos interculturales en el Huasco Alto. En Dursten, J. (Ed.). Pueblos originarios y sociedad nacional en Chile. Programa de las Naciones Unidas para el Desarrollo (PNUD), Santiago de Chile: PNUD, 115-127.

Nazer, R. (2004). Nacionalización y Privatización del cobre chileno 1971-2002. En Pensamiento Crítico - Revista Electrónica de Historia, 4, en: http://www.archivochile. com/Ideas_Autores/pintoj/pintoj0007.pdf

Nem Singh, J. (2010). Reconstituting the Neostructuralist State: the Political Economy of Continuity and Change in Chilean Mining Policy, Third World Quarterly, 31(8), 1413-1433.

Observatorio Latinoamericano de Conflictos Ambientales (OLCA) (2011). Minería al límite. Análisis de tres casos de minería de frontera en América Latina. Santiago de Chile: Equipo OLCA.

Observatorio Latinoamericano de Conflictos Ambientales (OLCA) (2012). Empresas extractivistas en los territorios. Juego de máscaras que hay que descifrar. Santiago de Chile: Equipo OLCA.

Perreault, T. \& Valdivia, G. (2010). Hydrocarbons, Popular Protest and National Imaginaries: Ecuador and Bolivia in Comparative Context. Geoforum, 41(4), 689-699.

Pratt, B. (2012). The Camisea Gas Project: Indigenous Social Movements and International NGOs in the Peruvian Amazon. En Bebbington, A. (Coord.). Social Conflict, Economic Development and Extractive Industry. London: Routledge, 172-186. 
Prieto, M. (2014). Privatizing Water and Articulating Indigeneity: The Chilean Water Reforms and the Atacameño People (Likan Antai). Ph.D. diss., University of Arizona.

Revenue Watch Institute (2013). The 2013 Resource Governance Index. A Measure of Transparency and Accountability in the Oil, Gas and Mining Sector, see: http://www.resourcegovernance.org/sites/default/files/ rgi_2013_Eng.pdf (accessed 15.04.2016)

Rivera, C. (2011). Conflictos ambientales y redes transnacionales de defensa en el Chile postdictadura. Revista de Ciencias Sociales, 12(2), 231-246.

Romero Toledo, H., Romero Aravena, H. y Toledo Olivares, X. (2009). Agua, Poder y Discursos: Conflictos Socio-territoriales por la construcción de centrales hidroeléctricas en la Patagonia Chilena. Anuario de Estudios Americanos, 66(2), 81-103.

Salinas, B. (2007). Implicancias territoriales del conflicto Pascua Lama, Valle del Huasco, región de Atacama. Santiago: Universidad de Chile.

Schilling-Vacaflor, A. (2014). Rethinking the Link between Consultation and Conflict: Lessons from Bolivia's Gas Sector. Canadian Journal of Development Studies, 35(4), 503-521.

Schorr, B. (2014). Taming Contention in Extractive Conflicts: Corporate Strategies and Local Implications in the Valle del Huasco, Chile. Paper delivered for the XXXIII Conference of the Latin American Studies Association (LASA) in San Juan, Puerto Rico, May 26-30, 2015.

Silva, E. (2009). Challenging Neoliberalism in Latin America. Cambridge: Cambridge University Press.

Tarrow, S. \& Tilly, C. (2015). Contentious Politics (segunda edición revisada). Cambridge: Cambridge University Press.

Urkidi, L. (2010). A Glocal Environment Movement against Gold Mining: Pascua Lama in Chile. Ecological Economics, 70, 219-227.

Vergara, A. (2011). "Cuando el río suena, piedras trae”: Relaves de Cobre en la Bahía de Chañaral. Cuadernos de Historia, 35, 135-151.
Widener, P. (2007). Benefits and Burdens of Transnational Campaigns: A Comparison of Four Oil Struggles in Ecuador. Mobilization: An International Quarterly, 12(1), 21-36.

Yánez, N. y Molina, R. (2008). La gran minería y los derechos indigenas en el norte de Chile. Santiago: LOM.

Yánez, N. y Molina, R. (2011). Las aguas indigenas en Chile. Santiago: LOM. 